\title{
A review of the quality and content of mobile apps to support lifestyle modifications following a transient ischaemic attack or 'minor' stroke
}

\author{
Seán R $0^{\prime}$ Connor ${ }^{1}$ (D), Frank Kee ${ }^{2}$, David R Thompson ${ }^{3}$ (D). \\ Margaret E Cupples ${ }^{2}$ (D) Michael Donnelly ${ }^{2}$ and Neil Heron ${ }^{2,4}$
}

Abstract

Objective: Secondary prevention is recommended to reduce cardiovascular risk after transient ischaemic attack (TIA) or 'minor' stroke. Mobile health interventions can provide accessible, cost-effective approaches to address modifiable risk factors, such as physical inactivity, hypertension and being overweight. The objective of this study was to evaluate the quality of apps for supporting lifestyle change following a TIA or 'minor' stroke.

Methods: Systematic searches of Google Play and the Apple Store were carried out to identify mobile apps released between 1 November 2019 and 1 October 2021. Keywords were used including stroke, TIA, lifestyle, prevention and recovery. Quality was assessed using the Mobile Application Rating Scale (MARS). Common components were identified with the Behaviour Change Technique (BCT) Taxonomy. Descriptive statistics were used to summarize the performance results for each app.

Results: Searches identified 2545 potential apps. Thirty remained after removing duplicates and screening titles and descriptions. Six were eligible after full review of their content. All apps included at least one BCT (range: 1-16 BCTs). The most frequent BCTs included 'information about health consequences' $(n=5 / 6)$, 'verbal or visual communication from a credible source' $(n=4 / 6)$ and 'action planning' $(n=4 / 6)$. The mean MARS score was $2.57 / 5$ (SD: 0.51 ; range: $1.78-3.36)$. No apps were of 'good' overall quality (scoring more than 4/5).

Conclusions: This is the first review of mobile health interventions for this population. Only a small number of apps were available. None were targeted specifically at people with a TIA or 'minor' stroke. Overall quality was low. Further work is needed to develop and test accessible, user designed, and evidence-informed digital interventions in this population.

\section{Keywords}

Mobile apps, mHealth, systematic review, transient ischaemic attack, 'minor' stroke, secondary prevention

\section{Introduction}

Acute cerebrovascular accident or stroke is a leading cause of morbidity and mortality. ${ }^{1}$ Stroke is frequently preceded by a history of transient ischaemic attack (TIA) or 'minor' stroke, with a first time TIA affecting approximately 50 in every 100,000 individuals. $^{2}$ In the absence of intervention, 7 and 90-day cardiovascular event risks of approximately $8 \%$ and $20 \%$ have been reported following a TIA. ${ }^{3,4}$ Recurrent stroke is more common after TIA

\footnotetext{
${ }^{1}$ School of Psychology, Queen's University Belfast, Belfast, UK ${ }^{2}$ Centre for Public Health, Queen's University Belfast, Belfast, UK ${ }^{3}$ School of Nursing and Midwifery, Queen's University Belfast, Belfast, UK ${ }^{4}$ School of Primary, Community and Social Care, Keele University, Staffordshire, UK

Corresponding author:

Neil Heron, Centre for Public Health, Queen's University Belfast, BT12 6BA, UK.

Email: n.heron@qub.ac.uk
} 
than an index stroke at various time intervals, with recurrence associated with delayed medical intervention. ${ }^{5}$ Evidence also indicates that TIAs may be associated with more prolonged functional and psychological effects than has previously been recognised. ${ }^{6}$ Early secondary preventative approaches are therefore essential to reduce stroke risk. ${ }^{7}$ These approaches include behavioural interventions to promote patient self-monitoring and reduction of cardiovascular risk factors such as arterial hypertension and physical inactivity. 8,9 Systematic reviews have, however, found limited evidence for the effectiveness of such interventions in terms of improvements in resting and peak systolic blood pressure, total cholesterol, aerobic capacity or cardiovascular event rate mortality. ${ }^{10,11}$ There is therefore currently insufficient evidence related to how to support behavioural interventions in TIA and 'minor' stroke populations. ${ }^{11}$

Access to well-developed, evidence-informed and highquality interventions in the early stages after a TIA can support secondary preventative and rehabilitative approaches aimed at reducing cardiovascular event and stroke recurrence risk. $^{12}$ This is important as patients may experience a range of different symptoms and might be anxious about the risk of recurrence, as well as reticent about initiating and maintaining changes in lifestyle. ${ }^{13}$

One potential method for the delivery of such interventions is via mobile or mHealth-based apps. These can provide widely accessible, cost-effective and adaptable tools to support self-management and lifestyle modification. ${ }^{14-17}$ Previous reviews evaluating mobile apps for supporting lifestyle modification in various clinical groups indicate that behavioural techniques including goal setting, performance monitoring and feedback are common features. These methods can be used alone or in combination and are defined as the smallest 'active' components of an intervention that can potentially influence or change behaviour. ${ }^{18}$ mHealth interventions can also provide access to evidence-based health information and incorporate adaptive methods including suggestions, prompts, reminders and motivational messages, delivered via notifications included within the apps. Interventions delivered in this manner may be more effective in helping to alter determinants of individual's beliefs and behaviours when compared with other methods of delivery. ${ }^{19}$ There is also evidence that mobile app based-interventions can be associated with increased user engagement and have more effective outcomes in comparison to other forms of supported selfmanagement. ${ }^{19}$ However, while uptake of mHealth interventions in the short term is often acceptable, many patients do not continue to use apps for a variety of reasons including usability issues, lack of time, or due to them not being seen as relevant to individual needs. ${ }^{20}$ Although the development and use of mobile apps for supporting patient self-management in various health conditions continues to increase, some studies have reported that apps frequently have limitations in the rigour of their development and the accuracy of the clinical information provided. ${ }^{21,22}$

Particularly in the early phase after a TIA or 'minor' stroke, high-quality, evidence-informed mobile apps, may provide valuable secondary preventative approaches to help reduce cardiovascular risk factors such as physical inactivity, hypertension, and being overweight. However, no previous studies have examined relevant apps in this population. Therefore, the primary objective of this study was to evaluate the quality of publically accessible mobile apps that aim to support lifestyle changes following a TIA or 'minor' stroke.

\section{Methods}

The review process was conducted according to the Preferred Reporting Items for Systematic reviews Meta-Analyses guidelines. ${ }^{23}$

\section{Search strategy}

Systematic searches of the Google Play Store and Apple App Store were carried out to identify potentially relevant mobile apps released between 1 November 2019 and 1 October 2021. Searches were carried out by two independent reviewers (SOC and NH) using of the following terms: 'Stroke', 'transient ischaemic attack', 'lifestyle', 'exercise', 'physical activity', 'diet', 'prevention', 'secondary prevention', 're-occurrence', 'recovery' and 'rehabilitation'. Examples of search term combinations included: 'Stroke', plus 'prevention'; and 'Stroke', plus 'recovery', plus 'rehabilitation'. Searches were limited to mobile apps in the Apple and Google Play stores to exclude information provided in books or other formats. No other search limitations were applied.

\section{App inclusion criteria and selection process}

To meet the inclusion criteria, apps were required to contain information on TIA, 'minor' stroke or stroke risk reduction and at least one component aimed at addressing modifiable risk factors including physical inactivity, hypertension, smoking, diet or being overweight. Apps were excluded if they were designed exclusively as aids to stroke recognition or diagnosis; were aimed at providing specific rehabilitation including arm movement recovery, speech or communication therapy; were not freely available to access within the United Kingdom or were not written in or translatable to English. Any disagreements on inclusion were resolved by discussion and consensus decisions reached between reviewers. The titles and descriptions of each app identified during the searches were initially examined by both independent reviewers 
and potentially suitable apps were then assessed in full to confirm eligibility. To assess apps, they were downloaded to either an Android (Motorola G8 power) or iPhone (iPhone 8). If apps were available in both app stores, the android version was assessed. Prior to data extraction each included app was used on at least three occasions and for a total of at least $25 \mathrm{~min}$ in order to become familiar with it and to fully utilise its functions and adequately review the content.

\section{Data extraction}

Data extraction was conducted using a pre-defined Excel form. This form was piloted on four non-related apps prior to commencing the review to ensure standardised methods of recording. Two reviewers (SOC and $\mathrm{NH}$ ) independently extracted data from the included apps. This included: app name, developer name and affiliation, date of last update, source (Google Play or Apple App Store), stated intended population, behaviours targeted (e.g. physical activity, blood pressure monitoring, diet, medication adherence) and app functions (e.g. notifications, data entry).

\section{Assessment of app quality}

Quality was assessed using the Mobile Application Rating Scale (MARS). ${ }^{24}$ This scale has been widely used $^{24-28}$ and has acceptable reliability and validity. ${ }^{23,25}$ The scale includes 19 items grouped in four domains: 1. Engagement (five items assessing the extent to which the app engages target users); 2. Functionality (four items assessing how easy the app is to navigate and the overall app performance); 3. Aesthetics (three items assessing visual appearance and style); and 4. Information (seven items assessing accuracy, quality and quantity of the app). Individual items are rated on a 1 to 5 -point ordinal scale. A score of between 1 and $2 / 5$ is considered as 'poor' quality, while $3 / 5$ is 'acceptable' and at least $4 / 5$ is 'good' quality. $^{23}$ The MARS also includes a separate, 'subjective' quality section (consisting of four items). Prior to conducting the quality assessment, reviewers met to discuss use of the MARS to ensure standardised methods of recording. The tool was also piloted on four non-related apps prior to assessment. Both reviewers (SOC and $\mathrm{NH}$ ) then independently rated the included apps. Where scores differed by a single point, the mean of the two ratings was used, with any scores differing by more than a single point being resolved through discussion and consensus agreement between reviewers. Mean scores were calculated for each domain and an overall quality score was calculated based on the aggregated mean values for each of the four domains. The mean score for subjective quality was also calculated.

\section{Identification of Behaviour Change Techniques in included apps}

Behavioural characteristics of the included apps were independently coded by two reviewers (SOC and $\mathrm{NH}$ ) using the structured Behaviour Change Technique (BCT) Taxonomy (Version 1.0 $)^{29}$ which consists of 93 hierarchically clustered items. Both reviewers were trained in BCT coding and had previous experience in using the taxonomy to assess digital interventions. Coding was conducted using the same pre-defined Excel form used for data extraction. Pilot testing was used to ensure standardisation of coding prior to independent assessment of each app. Scores of ' 0 ' for absent or ' 1 ' for present were given for each BCT, with higher scores indicating the presence of a more substantial behavioural component to the app.

\section{Statistical analyses}

Descriptive data are presented on the frequency of BCTs in each app. Means, standard deviations and range values for each MARS domain, as well as the overall quality scores and subjective quality scores, were calculated. Level of agreement prior to consensus between independent raters for BCT coding and MARS ratings was determined using weighted kappa's $(k)$ and intraclass correlation coefficients. These were calculated using MedCalc [MedCalc Software, Ostend, Belgium] and SPSS: Version 25 [Armonk, NY: IBM Corp]. Level of agreement was determined based on the following criteria: less than 0.60 - poor to fair agreement; 0.61 to 0.80 - strong agreement and 0.81 or more near complete agreement. ${ }^{30}$

\section{Results}

\section{App selection}

A total of 2543 potential apps were identified. Following removal of duplicates and screening of app titles and descriptions, 30 were downloaded and assessed in full to check against the study inclusion criteria. Four potential apps were not available in the United Kingdom. Of the apps screened in full, six were eligible for inclusion (see Figure 1). All were available from Google Play and the Apple app stores and were freely available to download.

\section{Characteristics and included Behaviour Change Techniques}

Two apps required a login to be set up prior to use and both used push notifications or reminders. None were targeted specifically at patients with a TIA but all included relevant information and support after a TIA or 'minor' stroke alongside general information on stroke or its prevention. Four apps included diet and physical activity or exercise 


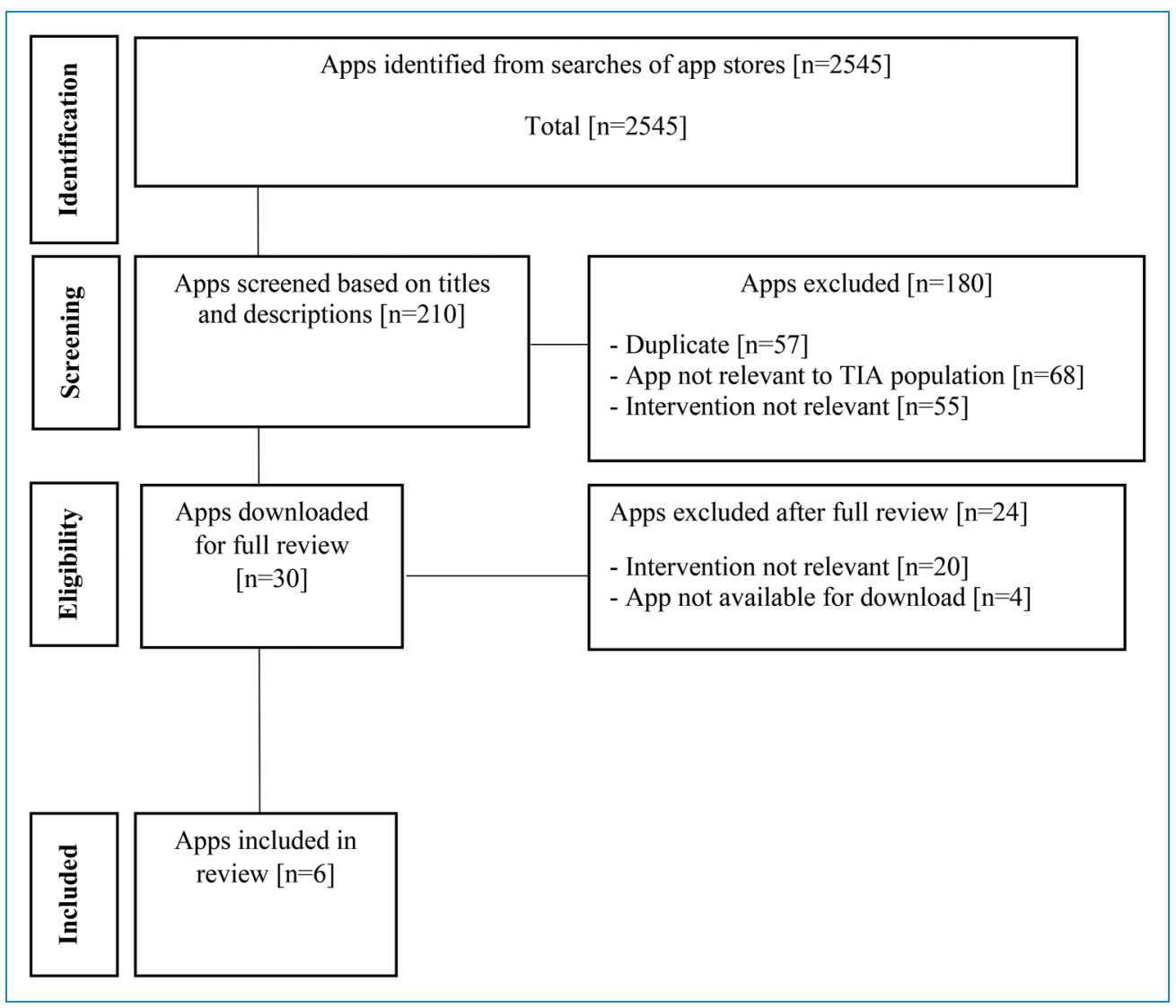

Figure 1. Flow chart showing app selection process.

advice and support, while two were targeted at physical activity or exercise only. All included information on stroke symptom recognition based on the FAST mnemonic (Facial weakness, Arm weakness, Speech problems, Time to call emergency services). Two also included a selfcompleted stroke risk assessment tool. Two included audiovisual materials with embedded videos and two included data entry methods such as tracking of physical activity in the form of manual step count entry. Characteristics of the apps are summarised in Table 1. Twenty-four different BCTs were identified. There was an average of 6.8 BCTs (ranging from 1 to $17 \mathrm{BCTs}$ ). The most common BCTs were 'information about health consequences' (5/6), verbal or visual communication from a 'credible source'

Table 1. Characteristics of the included apps.

\begin{tabular}{|c|c|c|c|c|c|c|}
\hline App name & Version & $\begin{array}{l}\text { Date of last } \\
\text { update }\end{array}$ & System & $\begin{array}{l}\text { Notifications/ } \\
\text { reminders }\end{array}$ & $\begin{array}{l}\text { Audio-visual material } \\
\text { included }\end{array}$ & $\begin{array}{c}\text { Number of } \\
\text { BCTs }\end{array}$ \\
\hline Hope after stroke & 3.1 .0 & August 2021 & Android/iOS & No & No & 7 \\
\hline $\begin{array}{l}\text { Stroke, causes, symptoms, } \\
\text { treatment }\end{array}$ & 9.0 & October 2019 & Android/iOS & No & No & 1 \\
\hline Strokemark & 1.0 .0 & July 2019 & Android/iOS & Yes & Yes & 17 \\
\hline Strocit plus & 1.0 .4 & October 2016 & Android/iOS & No & No & 1 \\
\hline How to prevent stroke & 1.0 .0 & November 2019 & Android/iOS & No & No & 6 \\
\hline Stroke Riskometer & 1.1 & August 2021 & Android/iOS & Yes & Yes & 9 \\
\hline
\end{tabular}

BCT: Behaviour Change Technique. 
(4/6) and 'action planning' (4/6) (see Table 2). The two apps with the most BCTs were 'Strokemark' $(n=17)$ and 'Stroke Riskometer' $(n=9)$.

Table 2. Number of apps with specific Behaviour Change Techniques included as components.

\begin{tabular}{|l|l|}
\hline Behaviour change technique & $\begin{array}{c}\text { Frequency } \\
(\%)\end{array}$ \\
\hline 5.1. Information about health consequences & 5 \\
\hline 9.1. Credible source & 4 \\
\hline 1.4. Action planning & 2 \\
\hline 8.1. Behavioural practice/rehearsal & 2 \\
\hline 3.1. Social support (unspecified) & 2 \\
\hline 1.1.Goal setting (behaviour) & 2 \\
\hline \begin{tabular}{l} 
1.3. Goal setting (outcome) \\
\hline 4.1. Instruction on how to perform the \\
behaviour
\end{tabular} & 3 \\
\hline
\end{tabular}

8.2. Behaviour substitution

11.1. Pharmacological support

1.2. Problem solving

3.3. Social support (emotional)

2.3. Self-monitoring of behaviour

8.7. Graded tasks

10.4. Social reward

5.4. Monitoring of emotional consequences

1.6. Discrepancy between current behaviour and goal

1.5. Review behaviour goal(s)

1

1.9. Commitment

1

6.1. Demonstration of the behaviour

1

7.1. Prompts/cues

1

1.7. Review outcome goal(s)

1

8.4. Habit reversal

1

12.1. Restructuring the physical environment

\section{Quality assessment}

Mean overall quality score assessed using the MARS was 2.58/5 (SD: 0.51 ) with scores ranging from 1.78 to 3.36 (see Table 3). Of the four domains assessed, 'Functionality' had the highest mean score (3.25/5; SD: $0.29)$ with 'Engagement' having the lowest score (2.12/5; SD: 0.81). No apps were rated as 'good' (a score of at least $4 / 5$ on the MARS overall quality score). Mean subjective quality scores were substantially lower than the overall quality scores $(2.03 / 5$; SD: 0.77$)$ and showed greater variation between apps with scores ranging from 1.5 to 3.26. A planned analysis to examine the relationship between the number of BCTs included and the overall app quality scores using Spearman's correlation coefficient was not possible due to the small number of included apps. However, the most behavioural components were found in the two highest quality apps ('Strokemark' and 'Stroke Riskometer'), which had respective aggregated scores (calculated based on the mean values $/ 5$ for each domain) of 3.36/5 (SD: 0.41) and 3.13/5 (SD: 0. 42).

\section{Inter-rater reliability of measurements}

MARS scores were within a single point for all ratings and good levels of agreement between independent ratings prior to consensus were observed for both the BCT codings and MARS scores (all were between 0.78 and 0.94 , indicting strong agreement. ${ }^{30}$ )

\section{Discussion}

\section{Principal findings}

The aim of this review was to evaluate the quality of mobile apps to support lifestyle modification after TIA or 'minor' stroke. Only six apps relevant to this clinical context were identified. None were targeted specifically at patients with a TIA only but included information for people with a TIA as part of apps providing general information on stroke or its prevention. The apps were primarily aimed at providing advice to support lifestyle changes intended to reduce stroke risk. Approximately seven BCTs were identified in each app. The overall quality of the apps varied and was relatively low, with none being rated as 'good' according to the MARS scoring criteria. The lowest mean scores were for the 'Engagement' domain of the MARS with higher scores observed for 'Functionality'. These findings indicate that the apps were generally better designed in terms of their usability but may have lacked behavioral and design features associated with sustained usage.

The two apps with the highest quality scores ('Strokemark' and 'Stroke Riskometer') included the highest number of behavioural techniques. This finding reflects those of other reviews exploring the quality of 
Table 3. Mean Mobile Application Rating Scale scores for each app.

\begin{tabular}{|c|c|c|c|c|c|c|}
\hline App name & $\begin{array}{l}\text { Engagement } \\
\text { score/5 }\end{array}$ & $\begin{array}{l}\text { Functionality } \\
\text { score/5 }\end{array}$ & $\begin{array}{l}\text { Aesthetics } \\
\text { score /5 }\end{array}$ & $\begin{array}{l}\text { Information } \\
\text { score/5 }\end{array}$ & $\begin{array}{l}\text { Overall mean } \\
\text { score } / 5^{\star}(S D)\end{array}$ & $\begin{array}{l}\text { Subjective } \\
\text { score/5 }\end{array}$ \\
\hline Hope after stroke & 1.80 & 3.66 & 3.33 & 2.16 & $2.74(0.89)$ & 2.00 \\
\hline $\begin{array}{l}\text { Stroke, causes, } \\
\text { symptoms, treatment }\end{array}$ & 1.20 & 3.00 & 1.33 & 1.60 & $1.78(0.17)$ & 1.50 \\
\hline Strokemark & 3.80 & 3.50 & 3.33 & 2.83 & $3.36(0.41)$ & 3.26 \\
\hline Strocit plus & 1.60 & 3.00 & 2.33 & 2.60 & $2.38(0.63)$ & 1.25 \\
\hline How to prevent stroke & 1.20 & 3.33 & 2.33 & 1.60 & $2.11(0.50)$ & 2.25 \\
\hline Stroke Riskometer & 3.20 & 3.00 & 3.00 & 3.33 & $3.13(0.43)$ & 1.96 \\
\hline Mean score (SD) & 2.22 (SD: 0.81) & 3.25 (SD: 0.29) & 2.61 (SD: 0.67) & 2.35 (SD: 0.79) & $2.58(0.51)$ & $2.03(0.77)$ \\
\hline
\end{tabular}

${ }^{\star}$ Calculated based on a mean aggregated score/5 across the four domains (Engagement, Functionality, Aesthetics and Information).

apps in different health conditions and populations that have confirmed an association between the number of BCTs and reported app quality. ${ }^{31-33}$ Although mHealth interventions can provide access to evidence-based health information, delivery of information by itself without further behaviour support might not be sufficient to initiate behavioural changes. ${ }^{34,35}$ Both of the better quality apps in this review included BCTs such as self-monitoring which may be associated with increased user engagement. Other adaptive methods contained in these apps, and which may be effective at promoting behavioural change, included prompts or reminders delivered via notifications. ${ }^{36,37}$ Although the number of BCTs identified in the apps is in line with those reported in previous reviews of apps aimed at promoting lifestyle changes, ${ }^{33,38}$ it is uncertain which number and combination of behaviour interventions are the most effective for promoting lifestyle changes ${ }^{39,40}$ and this requires further investigation.

Although the behavioural components of the apps in this review commonly included health information and advice, other studies exploring health apps have typically reported self-monitoring and goal setting as more frequent intervention components. ${ }^{33,35,41}$ However, only two apps in this review included advice on action planning, and this was supported with additional advice on goal setting or selfmonitoring of behaviour in only one app. These elements may be especially important in resources designed for use in the early phases following a TIA, when users may require support to initiate graduated lifestyle changes. ${ }^{42,43}$ Although some behavioural methods, such as providing health information, may be readily self-delivered and therefore suitable for delivery as part of mHealth enabled selfmanagement approaches, others may be contingent on the format of intervention delivery. For example, behavioural approaches such as goal setting involve reflective or deliberative processes, and users' sense of competence may be affected when goals set as part of an online only intervention are unmet. ${ }^{44,45}$ There is therefore currently limited evidence for the acceptability and efficacy of these techniques when behavioural approaches are included as part of mHealth interventions and they are required to be self-enacted.

This review highlights a number of issues in terms of the availability of suitable apps for a TIA population. Firstly, apps for supporting people after a TIA are limited in number and are not easy to locate (requiring potential users to access apps designed principally for people with more significant stroke). Much of the information included in such apps may therefore not be specifically tailored to the needs of people with a TIA or 'minor' stroke. Available apps are of generally low to moderate quality only and include few evidence-based behavioural components. There are subsequently little data on the core components of mHealth interventions in this population that are associated with increased engagement and usage needed for sustained behavioural changes. ${ }^{46}$ These components might include greater use of goal setting techniques, recognition and reward systems, and use of self-entered or automated data entry. More adaptive components could also include tailored reminders or prompts, motivational messages and content which is responsive to inputted data. ${ }^{47-49}$ It should be acknowledged, however, that behavioural theories have not hitherto adequately accounted for dynamic relationships and feedback between behavioural determinants and intervention components. ${ }^{50}$

\section{Strengths and limitations}

This review has a number of key strengths including a comprehensive and systematic search strategy, with apps 
identified within publically accessible app stores. No limits were applied based on app store ratings or number of downloads as it was felt this could create bias in favour of commercially sponsored apps. Independent data extraction and standardised quality assessment procedures using the MARS were employed and strong inter-rater reliability of these measures was found. There are limitations that must be considered when interpreting the findings of this review. Only a small number of apps were identified and due to restrictions placed on some apps, these were only available within the country where they were developed and were not accessible for download within the United Kingdom. Another limitation is that people with a history of TIA were not included in the assessment of apps. This could have improved the strength of the conclusions made within the review and their meaning and relevance for this population. Findings are also based only on publically accessible apps available from app stores, and any previously, or currently being developed as part of research studies were not included. This may have limited the number of apps included in the review, and the conclusions drawn on the quality of apps for individuals with a history of TIA.

\section{Conclusions}

This is the first systematic review of mHealth interventions for a TIA or 'minor' stroke population. As the use of mHealth interventions to support patient self-management increases, it is important that well-developed, high-quality apps are made available. This is important as mHealth interventions can be widely accessible and can help to promote secondary prevention approaches. It is essential that apps provide accurate clinical information and include features to ensure they have good usability and are engaging. However, findings suggest that apps that exist to support lifestyle modifications may not meet the needs of people with a TIA. Apps were found to be of low overall quality and although they were found to have better quality scores in terms of their 'functionality', scores were lowest for 'engagement'. Although the apps did include some behavioural components, the most common technique was health information provision which would not be sufficient to effect behavioural change by itself. Additional work is required to develop specific, engaging, evidence-based and theory-informed mHealth interventions for TIA or 'minor' stroke populations. This work should explore user perspectives on usability and usefulness and examine which combinations of self-enactable behavioural components are most effective at improving important outcomes in this population. Further studies will be conducted to develop and test an mHealth intervention for use in the early phase after TIA or 'minor' stroke. The findings of this review will be used to inform a behavioural analysis based on relevant underpinning theory and evidence from other previous studies, as well as to develop a proposed logic model intended to represent key factors that would potentially mediate outcomes of this planned intervention.

Conflict of interest: The author(s) declared no potential conflicts of interest with respect to the research, authorship, and/or publication of this article.

Contributorship: Seán O'Connor contributed to the study design, search strategy, screening and data extraction, and analysis of results and drafting of the manuscript. Frank Kee, David Thompson, Margaret Cupples and Michael Donnelly contributed to the conception and development of the original research question, the study design, interpretation of results and critically reviewed and revised the manuscript. Neil Heron contributed to the conception and development of the original research question, the study design, search strategy, screening and data extraction, analysis and interpretation of results, and drafting, critically reviewing and revising the manuscript. All authors approved the final version of the manuscript.

Ethical approval: Not applicable; no human subjects

Funding: The author(s) disclosed receipt of the following financial support for the research, authorship, and/or publication of this article: This research is supported by funding received from Northern Ireland Chest, Heart \& Stroke (NICHS) and from the National Institute of Health Research (NIHR) Clinical Lectureship for Dr Neil Heron.

\section{Guarantor: SOC}

ORCID iDs: David R Thompson (D) https://orcid.org/0000-00018518-6307

Margaret E Cupples (iD https://orcid.org/0000-0002-4248-9700

Seán R O'Connor (iD https://orcid.org/0000-0001-6805-8899

\section{References}

1. GBD 2016 Neurological Disorders Collaborator Group. Global, regional, and national burden of neurological disorders during 1990-2016: a systematic analysis for the Global Burden of Disease Study 2016. Lancet Neurol 2019; 18: 459-480.

2. Torres Macho J, Peña Lillo G, Pérez Martínez D, et al. Outcomes of atherothrombotic transient ischemic attack and minor stroke in an emergency department: results of an outpatient management program. Ann Emerg Med 2011; 57: 510-516.

3. Giles MF and Rothwell PM. Risk of stroke early after transient ischaemic attack: a systematic review and meta-analysis. Lancet Neurol 2007; 6: 1063-1072.

4. Kamal N, Hill MD, Blacquiere DP, et al. Rapid assessment and treatment of transient ischemic attacks and minor stroke in Canadian Emergency Departments: time for a paradigm shift. Stroke 2015; 46: 2987-2990.

5. Akijian L, Ní Chróinín D, Callaly E, et al. Why do transient ischemic attack patients have higher early stroke recurrence risk than those with ischemic stroke? Influence of patient 
behavior and other risk factors in the North Dublin Population Stroke Study. Int J Stroke 2017; 12: 96-104.

6. Liao XL, Zuo LJ, Zhang N, et al. Impairment of Cognition and sleep quality for patients after acute ischemic stroke or transient ischemic attack (ICONS) Investigators. The occurrence and longitudinal changes of cognitive impairment after acute ischemic stroke. Neuropsychiatr Dis Treat 2020; 26: 807-814.

7. Diener HC and Hankey GJ. Primary and secondary prevention of ischemic stroke and cerebral hemorrhage: JACC focus seminar. J Am Coll Cardiol 2020; 75: 1804-1818.

8. Ahmadi M, Laumeier I, Ihl T, et al. A support programme for secondary prevention in patients with transient ischaemic attack and minor stroke (INSPiRE-TMS): an open-label, randomised controlled trial. Lancet Neurol 2020; 19: 49-60.

9. Zheng $\mathrm{S}$ and Yao B. Impact of risk factors for recurrence after the first ischemic stroke in adults: a systematic review and meta-analysis. J Clin Neurosci 2019; 60: 24-30.

10. Deijle IA, Van Schaik SM, Van Wegen EE, et al. Lifestyle interventions to prevent cardiovascular events after stroke and transient ischemic attack: systematic review and meta-analysis. Stroke 2017; 48: 174-179.

11. Heron N, Kee F, Cardwell C, et al. Secondary prevention lifestyle interventions initiated within 90 days after TIA or 'minor' stroke: a systematic review and meta-analysis of rehabilitation programmes. Br J Gen Pract 2017; 67: e57-e66.

12. Seo WK, Kang J, Jeon M, et al. Feasibility of using a mobile application for the monitoring and management of stroke-associated risk factors. J Clin Neurol 2015; 11: 142-148.

13. Lam KH, Blom E and Kwa VIH. Predictors of quality of life 1 year after minor stroke or TIA: a prospective single-centre cohort study. BMJ Open 2019; 9: e029697.

14. Coorey GM, Neubeck L, Mulley J, et al. Effectiveness, acceptability and usefulness of mobile applications for cardiovascular disease self-management: systematic review with meta-synthesis of quantitative and qualitative data. Eur $J$ Prev Cardiol 2018; 25: 505-521.

15. Willeit P, Toell T, Boehme C, et al. STROKE-CARD study group. STROKE-CARD care to prevent cardiovascular events and improve quality of life after acute ischaemic stroke or TIA: A randomised clinical trial. EClinicalMedicine 2020;25:100476.

16. Patomella AH, Farias L, Eriksson C, et al. Engagement in everyday activities for prevention of stroke: feasibility of an mHealth-supported program for people with TIA. Healthcare (Basel) 2021; 9: 968.

17. Heron N, O'Connor SR, Kee F, et al. Development of a digital lifestyle modification intervention for use after transient ischaemic attack or minor stroke: a person-based approach. Int J Environ Res Public Health 2021; 18: 4861.

18. Sheeran P, Klein WM and Rothman AJ. Health behavior change: moving from observation to intervention. Annu Rev Psychol 2017; 68: 573-600.

19. Plow $M$ and Golding M. Using mHealth technology in a selfmanagement intervention to promote physical activity among adults with chronic disabling conditions: randomized controlled trial. JMIR Mhealth Uhealth 2017; 5: e185.

20. Yardley L, Spring BJ, Riper H, et al. Understanding and promoting effective engagement with digital behavior change interventions. Am J Prev Med 2016; 51: 833-842.
21. Byambasuren O, Sanders S, Beller E, et al. Prescribable mHealth apps identified from an overview of systematic reviews. NPJ Digit Med 2018; 1: 12.

22. Llorens-Vernet $\mathrm{P}$ and Miró J. Standards for mobile health-related apps: systematic review and development of a guide. JMIR Mhealth Uhealth 2020; 8: e13057.

23. Liberati A, Altman DG, Tetzlaff J, et al. The PRISMA statement for reporting systematic reviews and meta-analyses of studies that evaluate health care interventions: explanation and elaboration. Br Med J 2009; 2009: b2700.

24. Stoyanov SR, Hides L, Kavanagh DJ, et al. Mobile app rating scale: a new tool for assessing the quality of health mobile apps. JMIR Mhealth Uhealth 2015; 3: e27.

25. Kim BY, Sharafoddini A, Tran N, et al. Consumer mobile apps for potential drug-drug interaction check: systematic review and content analysis using the Mobile App Rating Scale (MARS). JMIR Mhealth Uhealth. 2018; 6: e74.

26. Siddique AB, Krebs M, Alvarez S, et al. Mobile apps for the care management of chronic kidney and end-stage renal diseases: systematic search in app stores and evaluation. JMIR Mhealth Uhealth 2019; 7: e12604.

27. Martín Payo R, Harris J and Armes J. Prescribing fitness apps for people with cancer: a preliminary assessment of content and quality of commercially available apps. J Cancer Surviv 2019; 13: 397-405.

28. Devan H, Farmery D, Peebles L, et al. Evaluation of selfmanagement support functions in apps for people with persistent pain: systematic review. JMIR Mhealth Uhealth 2019; 7: e13080.

29. Domnich A, Arata L, Amicizia D, et al. Development and validation of the Italian version of the Mobile Application Rating Scale and its generalisability to apps targeting primary prevention. BMC Med Inform Decis Mak 2016; 16: 83.

30. Michie S, Wood CE, Johnston M, et al. Behaviour Change Techniques: the development and evaluation of a taxonomic method for reporting and describing behaviour change interventions (a suite of five studies involving consensus methods, randomised controlled trials and analysis of qualitative data). Health Technol Assess 2015; 19: 1-188.

31. Viera AJ and Garrett JM. Understanding interobserver agreement: the kappa statistic. Fam Med 2005; 37: 360-363.

32. Schoeppe S, Alley S, Rebar AL, et al. Apps to improve diet, physical activity and sedentary behaviour in children and adolescents: a review of quality, features and Behaviour Change Techniques. Int J Behav Nutr Phys Act 2017; 14: 83.

33. Wang Y, Wang Y, Greene B, et al. An analysis and evaluation of quality and behavioral change techniques among physical activity apps in China. Int J Med Inform 2020; 133: 104029.

34. Bardus M, van Beurden SB, Smith JR, et al. A review and content analysis of engagement, functionality, aesthetics, information quality, and change techniques in the most popular commercial apps for weight management. Int $J$ Behav Nutr Phys Act 2016; 13: 35.

35. Hendrickx W, Vlietstra L, Valkenet K, et al. General lifestyle interventions on their own seem insufficient to improve the level of physical activity after stroke or TIA: a systematic review. BMC Neurol 2020; 1: 168.

36. Connell LE, Carey RN, de Bruin M, et al. Links between behavior change techniques and mechanisms of action: an expert consensus study. Ann Behav Med 2019; 53: 708-720. 
37. Alkhaldi G, Hamilton FL, Lau R, et al. The effectiveness of prompts to promote engagement with digital interventions: a systematic review. J Med Internet Res 2016; 18: e6.

38. Morrison LG, Hargood C, Pejovic V, et al. The effect of timing and frequency of push notifications on usage of a smartphone-based stress management intervention: an exploratory trial. PLoS One 2017; 12: e0169162.

39. Alessa T, Hawley MS, Hock ES, et al. Smartphone apps to support self-management of hypertension: review and content analysis. JMIR Mhealth Uhealth 2019; 7: e13645.

40. Knittle K, Heino M, Marques MM, et al. The compendium of self-enactable techniques to change and self-manage motivation and behaviour v.1.0. Nat Hum Behav 2020; 4: 215-223.

41. Whitehead L and Seaton P. The effectiveness of selfmanagement mobile phone and tablet apps in long-term condition management: a systematic review. J Med Internet Res 2016; 18: e97.

42. Faiola A, Papautsky EL and Isola M. Empowering the aging with mobile health: a mHealth framework for supporting sustainable healthy lifestyle behavior. Curr Probl Cardiol 2019; 44: 232-266.

43. Kamoen O, Maqueda V, Yperzeele L, et al. Stroke coach: a pilot study of a personal digital coaching program for patients after ischemic stroke. Acta Neurol Belg 2020; 120: 91-97.
44. Crowfoot G, van der Riet P and Maguire J. Real-life experiences of people with transient ischaemic attack or minor stroke: a qualitative literature review. J Clin Nurs 2018; 27: 1381-1398.

45. Knittle K, Nurmi J, Crutzen R, et al. How can interventions increase motivation for physical activity? A systematic review and meta-analysis. Health Psychol Rev 2018; 12: 211-230.

46. Vandelanotte C, Kolt GS, Caperchione CM, et al. Effectiveness of a Web 2.0 intervention to increase physical activity in real-world settings: randomized ecological trial. $J$ Med Internet Res 2017; 19: e390.

47. Setiawan IMA, Zhou L, Alfikri Z, et al. An adaptive mobile health system to support self-management for persons with chronic conditions and disabilities: usability and feasibility studies. JMIR Form Res 2019; 3: e12982.

48. Liao P, Dempsey W, Sarker H, et al. Just-in-time but not too much: determining treatment timing in mobile health. Proc ACM Interact Mob Wearable Ubiquitous Technol 2018; 2: 179.

49. Forman EM, Goldstein SP, Crochiere RJ, et al. Randomized controlled trial of OnTrack, a just-in-time adaptive intervention designed to enhance weight loss. Transl Behav Med 2019; 9: 989-1001.

50. Riley WT, Rivera DE, Atienza AA, et al. Health behavior models in the age of mobile interventions: are our theories up to the task? Transl Behav Med 2011; 1: 53-71. 


\section{University Library}

\section{- M I I N E R VA \\ A gateway to Melbourne's research publications}

Minerva Access is the Institutional Repository of The University of Melbourne

Author/s:

O'Connor, SR;Kee, F;Thompson, DR;Cupples, ME;Donnelly, M;Heron, N

Title:

A review of the quality and content of mobile apps to support lifestyle modifications following a transient ischaemic attack or 'minor' stroke.

Date:

2021

Citation:

O'Connor, S. R., Kee, F., Thompson, D. R., Cupples, M. E., Donnelly, M. \& Heron, N. (2021). A review of the quality and content of mobile apps to support lifestyle modifications following a transient ischaemic attack or 'minor' stroke.. Digit Health, 7, pp.20552076211065271-. https://doi.org/10.1177/20552076211065271.

Persistent Link:

http://hdl.handle.net/11343/296046

License:

CC BY-NC-ND 